Analytical spectra in scientific documents

\author{
R. Manfre and L. Dumont
}

Integrating spectra from analytical instruments directly into scientific documents is a boon to scientific word processing. Software packages are now available for PC's and mini/mainframe computers.

\begin{abstract}
lin: number of scientific word processors (SWPs) available commercially has increased tremendously in the past five years. Programs are available for both mini/mainframes and personal computers (PCs). within a wide range of capabilities and prices. Scientists are becoming more aware of the usefulness of SWPS, as evidenced by the scores of articles in the literature comparing the attributes of various packages ". Scientists are also becoming

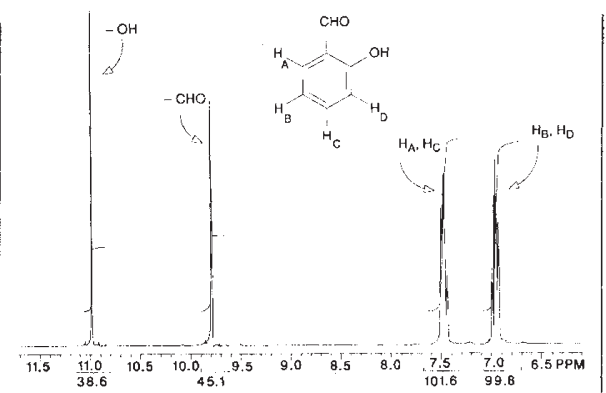
more demanding, more sophisticated uscrs of SWPS. These machines are becoming essential in modern laboratories, and scientists are looking toward the future to predict what further capabilities should he provided in the coming years ${ }^{4}$. The preparation of scientific documents using a computer is becoming standard.

Current scientific word processing programs perform many functions. Packages vary, but most SWPS allow researchers to write documents using full word processing functions; to use numerous fonts, such as Greek and European; and to create complex mathematical equations, molecules and reaction schemes, and tables and flow charts. Some also allow the incorporation of graphic images from other software packages, such as Lotus $1-2-3$ and $\mathrm{RS} / 1$, into the document being prepared. But many of the images that go into scientific reports, such as spectra and chromatograms, are created outside the SWP, making integration with other instruments essential to a full-function SWP . To enable the creation of complete documents, an SWP package should provide a means of including analytical images in reports by interfacing, either directly or indirectly, with laboratory instrumentation. Few packages available today provide this capability.

\section{Importing data}

At American Cyanamid chemists have used successfully a commerciallyavailable SWP package, ChemText, from Molecular Design Limited, to incorporate NMR spectra and HPLC chromatograms into documents created on a PC. The connection between the SWP on the PC and the NMR is direct, and involves one filetransfer step. In general, the method of transfer depends upon the hardware environment. In cases where the instrument is controlled by a PC as part of a laboratory information management system (LIMS), transfer will involve fewer steps; in other cases, it will involve more steps. The method used at American Cyanamid can be applied to any stand-alone instrument,
Fig. 1 An NMR file of salicylaldehyde from the Varian XL-300 Courtesy S. Alvarado.

such as an NMR, IR or HPLC, that supports Hewlett-Packard Graphic Language (HPGL) output, the language normally used to transfer information from an instrument to a Hewlett-Packard pen plotter.

The procedure to transfer information is fairly straightforward. To send information to the PC, a cable that would normally go from an RS-232 serial port on the instrument to a pen plotter is instead connected to an RS-232 serial port on the PC. Next, using a terminal emulator program, the chemist sets the configuration of the $\mathrm{PC}$ so that it is ready to receive data. This is done by matching communication parameters, such as baud rate, with the instrument and by setting the emulator to do an ASCII file capture. As a result, all subsequent information that is sent to the PC screen is saved to a file on the hard disk. When the chemist plots the spectrum from the instrument, the HPGL output scrolls across the screen on the PC and is saved to a file, instead of being sent to an HP plotter. The SWP package provides a utility function that converts the HPGL file format to a format it can read, allowing the spectrum to be displayed on the PC screen. The spectrum can then be manipulated on screen. It can be made larger or smaller, the pen style can be changed, and arrows, boxes or text can be added.

For example, the configuration of a Varian XL-300 NMR, which supports the HP7475 pen plotter for output, may be set to send HPGL files to a PC. To accomplish this, a 25-pin straight-through cable is run from the plotter port on the NMR to a serial port on the $\mathrm{PC}$, and the terminal emulator program is started and set to perform an ASCII file capture. The spectrum can then be plotted from the NMR as if the NMR were connected to an HP pen plotter. Once captured by the PC, the HPGL file is converted to a file format understood by the SWP, and read into the SWP for inclusion in a report. The spectrum may be enhanced on screen by inserting the chemical structure of the compound and indicating the proper chemical shift assignments (Fig. 1), and may then be inserted into a report.

A similar system may be set up to capture chromatograms from a SpectraPhysics $8800 \mathrm{HPLC}$, which stores its data on a Hewlett-Packard HP1000/3357 Laboratory Data System (LDS). Both scenarios are successful, but the SWP occasionally has difficulty incorporating very large files approaching a half megabyte.

An SWP for incorporating spectra directly from analytical instruments is also available for mini/mainframe computers. The MASS 11 system by Microsystems Engineering Corporation runs on a VAX, and is an integrated, modular package of office automation software that may include word processing and several other applications, including features for creating scientific documents. A recently released optional module allows the incorporation of graphics files, including HPGL files, into documents created on a VAX. With MASS11, an image captured from an analytical instrument may be scaled, trimmed and rotated, but other images may not be superimposed over it. Text can be made to wrap around the spectrum, if so desired, but the document portion of the program does not support bit mapping, so the image cannot be seen in the document until it is printed.

The two systems discussed allow complete scientific documents, including spectra and chromatograms from laboratory instruments that support HPGL output, to be created on a computer. In the future, competitive SWPs will recognize a greater variety of plot and print files. These programs will be able to import files created by scanners, allowing scientists to include photographs and paper-based spectral and chromatographic data in their reports. They will also be able to read files of digitized video stills, such as those from scanning electron microscopy work. In the near future, a scientist will be able to incorporate virtually any image needed in a document before it is printed.

Robert Manfre is at American Cyanamid Company, PO Box 400, Princeton, New Jersey 08540 USA, and Lise Dumont is at Molecular Design Limited, 2132 Farallon Drive, San Leandro, California 94577 USA. For more information, fill in reader service number 100 .

I. Marshall, J.C. J. Chem. Inf. Comput. Sci. 26, 87-92 (1986). 2. Love, R.A. \& Gerson, C.K. 1987 National Meeting of the American Chemical Society, Abstr. No. CINF 20.

. Dumont, L. Sci. comput. automat. 3, 16-24 (1987)

Dessy, R.E. Chemometrics Intell. Lab. Sys. 1, 309-320 (1987), Root, J.W. Computers Sci. 1, 79-82 (1987) 